\title{
The roles of perspective and language in children's ability to delay gratification
}

\author{
Caitlin E.V. Mahy ${ }^{\mathrm{a}, *}$, Louis J. Moses ${ }^{\mathrm{b}}$, Bronwyn O’Brien ${ }^{\mathrm{c}}$, Alex W. Castro ${ }^{\mathrm{c}}$, \\ Leia Kopp ${ }^{c}$, Cristina M. Atance ${ }^{\mathrm{c}}$ \\ a Department of Psychology, Brock University, St. Catharines, Ontario L2S 3A1, Canada \\ ${ }^{\mathrm{b}}$ School of Psychology, Victoria University of Wellington, Wellington 6140, New Zealand \\ ' School of Psychology, University of Ottawa, Ottawa, Ontario K1N 6N5, Canada
}

\section{A R T I C L E I N F O}

\section{Article history:}

Received 2 July 2019

Revised 18 November 2019

\section{Keywords:}

Delay of gratification

Self/other

Psychological distancing

Young children

Perspective-taking

Language

\begin{abstract}
A B S T R A C T
Increasing psychological distance is an established method for improving children's performance in a number of self-regulation tasks. For example, using a delay of gratification (DoG) task, Prencipe and Zelazo (Psychological Science, 2005, Vol. 16, pp. 501-505) showed that 3-year-olds delay more for "other" than they do for "self," whereas 4-year-olds make similar choices for self and other. However, to our knowledge, no work has manipulated language to increase psychological distance in children. In two experiments, we sought to manipulate psychological distance by replicating Prencipe and Zelazo's age-related findings and extending them to older children (Experiment 1) and also sought to manipulate psychological distance using the auxiliary verbs "want" and "should" to prime more impulsive preference-based decisions or more normative optimal decisions (Experiment 2). In Experiment 1, 96 3- to 7-year-olds showed age-related improvements and interactive effects between age and perspective on DoG performance. In Experiment 2, 132 3- to 7-year-olds showed age-related improvements and a marginal interaction between age and perspective on DoG performance, but no effect of auxiliary verbs was detected. Results are discussed in terms of differing developmental trajectories of DoG for self and other due to psychological distancing, and how taking another's perspective may boost DoG in younger children but not older children.
\end{abstract}

(c) 2019 Elsevier Inc. All rights reserved.

\footnotetext{
* Corresponding author.

E-mail address: caitlin.mahy@brocku.ca (C.E.V. Mahy).
} 


\section{Introduction}

Since Walter Mischel first developed the famous "marshmallow experiment" during the 1960s, interest in the development of children's delay of gratification (DoG), factors that affect its development, and the long-term outcomes that DoG predicts have remained steady. In fact, during the past few years there has been a particular increase in examining social and contextual factors that influence young children's ability to postpone gratification. For example, studies have documented that children's DoG is influenced by whether their in-group members also delay gratification (Doebel \& Munakata, 2018), by the trustworthiness of the experimenter (Michaelson \& Munakata, 2016), and by environmental reliability (Kidd, Palmeri, \& Aslin, 2013). Thus, although DoG paradigms were originally thought to be a relatively stable index of self-control (Mischel, Shoda, \& Rodriguez, 1989), recent work suggests that DoG performance is influenced by many factors, some of which are not due to stable individual differences between children (see Watts, Duncan, \& Quan, 2018). The goal of the current work was to examine the role of psychological distance in promoting young children's DoG behavior.

\section{DoG and developmental change}

Developmental work has shown that, compared with older preschoolers, young preschoolers tend to choose immediate smaller rewards rather than delaying gratification to receive larger rewards (Mischel et al., 1989; Moore, Barresi, \& Thompson, 1998; Prencipe \& Zelazo, 2005). What drives the development of DoG over the early childhood years? Inhibitory control is one possibility. In a DoG context, children must inhibit the desire for immediate rewards in order to delay for later rewards. Notably, inhibitory control undergoes significant developmental increases during early childhood (e.g., Zelazo, Carlson, \& Kesek, 2008) and, thus, might contribute to improvements in DoG (e.g., Moore et al., 1998). Theory of mind is another ability that might play a role in age-related increases in DoG during early childhood (e.g., Moore et al., 1998). Children must understand their current desires but also must understand that their own desires might change in the future and the consequences of this for emotional experience. For example, older children might be better able to consider their future desires and emotions such as regret (e.g., Weisberg \& Beck, 2010, 2012) and, therefore, may be less prone to temptation from immediate rewards. Indeed, Lemmon and Moore (2007) found that 4-year-olds' performance indicated that these children were aware of both immediate and future desires, whereas 3-year-olds' performance was based on immediate desires.

\section{Self-other differences in DoG}

Another factor that has been found to influence young children's DoG performance is whether the children are deciding for "self" versus "other." For example, Prencipe and Zelazo (2005) showed that 3-year-olds were more likely to delay rewards such as candies, stickers, and coins for the experimenter than for themselves, but by 4 years of age they delayed similarly for self and other, a point to which we return below. Recent work on children's future-oriented reasoning has also shown that preschoolers perform significantly better on certain tasks when asked to reason about another person's future compared with their own future-what we term an "other-over-self" advantage (Bélanger, Atance, Varghese, Nguyen, \& Vendetti, 2014; Russell, Alexis, \& Clayton, 2010). For instance, Bélanger et al. (2014) found that preschoolers were more likely to accurately predict that when another child was "all grown up" he would prefer coffee to Kool-Aid, for example, than when making the same prediction for themselves.

These findings are important for at least two reasons. From an applied perspective, they suggest that younger children's future-oriented decision making is optimized by asking these children to reason about another person rather than themselves. Theoretically, the findings suggest that in certain contexts children have the necessary conceptual understanding of the future but struggle to properly implement such knowledge when the prediction is about the self. If so, why might children have less difficulty in reasoning about another person's future as compared with their own future? 
Explanations for other-over-self advantage

According to Prencipe and Zelazo (2005), who drew on Barresi and Moore (1996) theory of perspective taking, young children have difficulty in delaying gratification for themselves because they adopt an exclusively first-person perspective on their behavior that leads to more impulsive choices (e.g., wanting to fulfill their immediate desires) and fail to consider that the delayed reward is the more rational choice. In contrast, they take a third-person or "outside" perspective for another person, leading to more rational decision making. As children develop, however, they are better able to take a third-person perspective on their own decision making or behavior (resulting in an increase in decisions to delay for self) and a first-person perspective on another's decision making or behavior (resulting in an increase in decisions to gratify another person's immediate desires or needs). Whereas for younger children this pattern results in response differences for self and other, for older children the pattern results in a more integrated perspective for self and other. In other words, older children seem to understand the benefits of delaying for both self and other but also seem to understand that sometimes both self and other have immediate desires to be fulfilled. The important point is that this response pattern is applied in an integrated fashion to both self and other, leading to fewer self-other differences in DoG. Indeed, as noted earlier, despite 3-year-olds making more delayed decisions for other versus self, Prencipe and Zelazo (2005) found that 4-year-olds' decisions for self and other were not significantly different.

Another variable that may be important in explaining younger children's differential responding for self and other is that when children think about their own future, their mental projections are heavily influenced by their current state (e.g., Atance \& Meltzoff, 2006; Kramer, Goldfarb, Tashjian, \& Lagattuta, 2017; Mahy, 2016; Mazachowsky, Koktavy, \& Mahy, 2019). In contrast, this influence is tempered when thinking about another person's future, leading to more optimal future-oriented choices (and an other-over-self advantage). Indeed, common to both DoG and Bélanger et al. (2014) preferences task is that they involve conflict between what is currently desirable (e.g., receiving one sticker immediately, preferring Kool-Aid) and what will be desirable in the future (e.g., receiving multiple stickers, preferring coffee). It may be that adopting the perspective of another person is especially beneficial in contexts where there is "present-future" conflict because this context reduces the inhibitory demands required to think about a future state in the presence of a conflicting current state. In other words, when thinking about another person's future, one's own current state is less of an "interfering" factor, leading to more accurate and objective reasoning.

This may be especially true of DoG tasks in which rewards are highly salient and immediately obtainable (Mischel et al., 1989; Prencipe \& Zelazo, 2005). Indeed, it might be easier for a child to realize the benefits of someone else versus oneself waiting to receive six candies because the child's immediate desire for one candy now may be highly salient and difficult to overcome. In line with this prediction, Moore et al. (1998) found that 3-year-olds' inhibition was positively related to choosing delayed versus immediate rewards for themselves.

\section{DoG and psychological distance}

Given that success in a DoG task requires children to overcome their current desires in order to focus on future rewards, it is plausible that manipulations that increase psychological distance would increase performance on such tasks. Taking a more psychologically distanced perspective may allow children to adopt a more objective stance on their desires by overcoming some of the demands that limit their DoG. Mischel's research suggests that decreasing the salience of the immediate reward during the delay (Mischel \& Ebbesen, 1970), encouraging distraction during the delay (Mischel, Ebbesen, \& Zeiss, 1972), and focusing on abstract qualities of the rewards (Mischel \& Baker, 1975; Mischel \& Moore, 1973) significantly improved children's DoG performance. These manipulations all focused on increasing psychological distance via altering the relative salience of the immediate reward and how it is conceptualized.

Importantly, only Prencipe and Zelazo (2005) study has directly examined the role of making DoG decisions for the self and another person (hence manipulating psychological distance by asking children to take different perspectives). Beyond the domain of DoG, increasing psychological distance by 
taking the perspective of another person has been shown to increase performance on tasks measuring cognitive control (White \& Carlson, 2016), episodic future thinking (Lee \& Atance, 2016; Mazachowsky et al., 2019; Russell et al., 2010), and perseverance (White et al., 2017). Thus, it seems that encouraging children to take the perspective of another person is an effective way to improve children's performance in a number of domains of development.

Another way to influence children's DoG is to use language to prime more desire- or rewardfocused behavior (resulting in less DoG) versus priming more rational optimal behavior to maximize rewards (resulting in more DoG). Some work suggests that priming children to focus on immediate or delayed rewards using language terms influences DoG performance. For example, Kesek, Cunningham, Packer, and Zelazo (2011) found that 4-year-old children's DoG behavior was influenced by implicit verbal information emphasizing either attaining immediate rewards or maximizing rewards in a story presented prior to the DoG task. Children made more decisions to delay when primed to maximize rewards and delayed less when primed to obtain immediate rewards. However, the study also found that explicit verbal instruction to "get the most rewards that you can" or to "get rewards as soon as you can" had no impact on children's delay behavior (Kesek et al., 2011). Taken together, this work suggests that children's DoG behavior might be influenced-at least implicitly-by verbal emphasis on either immediately obtaining or maximizing rewards.

Little work, however, has taken advantage of language in order to manipulate psychological distance. For example, even though children understand desire terms such as "want" around 3 years of age (e.g., Cassidy, 1998; Wellman \& Liu, 2004) and deontic terms such as "should" and "must" by 3 or 4 years of age (e.g., Cummins, 1996; Harris \& Nunez, 1996), no research has compared what children claim they "want" to do versus what they "should" do in a DoG context. In fact, Wellman and Miller (2008) argued that deontic reasoning is fundamental to theory of mind, suggesting that both understanding of obligation-permission and understanding of desire-belief develop early, are universally developing, and are inseparable. Despite this suggestion, there is no work examining the influence of children's understanding of desire- and deontic-based reasoning and its influence on DoG behavior.

Given children's early understanding of these desire (i.e., want) and deontic (i.e., should) terms, children might be able to distance themselves to think about what one should do because "should" may prime a more normative or optimal decision. In fact, Wellman and Miller (2008) argued that children's conceptions of obligation have an important impact on normative expectations. Thus, use of the word "should" might prime a sense of obligation, and thus a normative decision, in a DoG context. In contrast, having children focus on their desires might prime a more impulsive preference-based decision for the immediate reward.

\section{The current experiments}

Several studies show that during the early preschool years children make better future judgments and decisions for another person than for themselves (Bélanger et al., 2014; Prencipe \& Zelazo, 2005; Russell et al., 2010). It has been suggested, however, that after about 4 years of age self-other differences should disappear. Although Prencipe and Zelazo (2005) did indeed show that 3-year-olds benefitted from taking the perspective of another person, whereas 4-year-olds did not, the authors did not test older children; thus, nothing is known about self-other differences in DoG beyond 4 years. Determining this is important given that self-other differences may vary as a function of age. For example, young children who are closer to mastering a given task may benefit more from adopting the perspective of another person, as compared with older children whose performance is already high. As Barresi and Moore (1996) suggested (see also Prencipe \& Zelazo, 2005), integration between self and other increases over early childhood and children come to understand that other people have immediate desires that influence their decisions (ability to take a first-person perspective of others) and better understand that one's own decisions might be optimized by waiting for larger later rewards by taking a third-person perspective on the self. The rapid development of theory of mind and executive functioning during early childhood might drive some of the self-other differences observed. In all of these domains, taking a more distant perspective might help children's DoG by allowing the children to reason from a more detached perspective. As children get older, they may even make better decisions for 
self versus other in the context of DoG because decisions for self become more motivationally significant and because children may better identify with their future selves than with future others (e.g., Hershfield, 2011).

The main goal of Experiment 1 was to examine differences in DoG reasoning for self and other beyond 4 years of age. We included 3- to 7-year-old children because few studies have systematically examined DoG performance beyond 5 years despite both the theoretical and applied implications about what such findings might reveal. Children were given a choice delay task in which they chose between a smaller immediate reward and a larger later reward for both self and other (Prencipe \& Zelazo, 2005). Consistent with Prencipe and Zelazo (2005) findings, we expected that 3-year-olds would reason more optimally about another person's future than they would about their own future, whereas 4-year-olds' choices for self and other would be similar. Beyond 4 years, we expected that either choices for self and other would be similar or children would delay more for self versus other.

Experiment 2 sought to replicate findings of the first experiment but, in addition, examine the role of language. More specifically, half of the children were asked what they and the experimenter "wanted" to do and half were asked what they and the experimenter "should" do. We expected that children would perform better for both self and other when asked what they "should" do versus "wanted" to do. This language manipulation also serves to rule out a potential confound in Prencipe and Zelazo (2005) study; children in that study always were asked what they "wanted" to do and were asked what the experimenter "should" do. Thus, manipulating language served two goals in Experiment 2: (a) to examine whether using the auxiliary verb "should" versus "want" would improve performance in a choice delay task via increasing psychological distance and (b) to rule out the possibility that Prencipe and Zelazo (2005) self-other findings were due to using the auxiliary verbs "want" for self and "should" for other.

\section{Experiment 1}

\section{Method}

\section{Participants}

Participants were 96 typically developing children who participated in a larger study about children's future-oriented reasoning: 20 3-year-olds (11 girls; $M_{\text {age }}=42.80$ months, $S D=3.09$ ), 21 4-year-olds ( 12 girls; $M_{\text {age }}=52.71$ months, $S D=3.94$ ), 185 -year-olds ( 9 girls; $M_{\text {age }}=67.00$ months, $S D=3.01$ ), 19 6-year-olds (9 girls; $M_{\text {age }}=78.32$ months, $\left.S D=3.28\right)$, and 18 7-year-olds (9 girls; $M_{\text {age }}=$ 88.72 months, $S D=3.69)$. No participants were excluded. Children were predominantly White $(83.7 \%)$ and from middle-class backgrounds (67\% of parents held a university degree and $52 \%$ had household income over $\$ 75,000$ ). Children were recruited from participant databases at the University of Oregon and the University of Ottawa.

\section{Choice delay}

Children were told that they were going to play a "choices" game where they would get to choose between one reward now and a larger reward later. As in Prencipe and Zelazo (2005), the experimenter demonstrated two practice trials where she chose the immediate reward on the first trial and the delayed reward on the second trial while demonstrating the consequences of her choice. This was done so that children understood that either choice was acceptable. Children were then given a choice between receiving one reward (3 reward types: stickers, candies, or nickels) immediately, which they could eat or interact with, and receiving two, four, or six rewards later at the end of the study. If children chose to delay, the reward was placed in an opaque cup that was kept in children's view and given to them at the end of the session in $30 \mathrm{~min}$. Children made a choice for each reward type 3 times (one now vs. two later, one now vs. four later, and one now vs. six later) for self ("What do you want to do?") and three times for the experimenter ("What should I do?"), yielding 18 trials. Self and other blocks were counterbalanced such that half of the children received the self block followed by the other block and half received the other block followed by the self block. Within each block, each reward type and number was randomly selected until all 9 trial types were complete. Children were 
given a score out of 9 based on the number of times they chose to delay for themselves (self) and a score out of 9 for the number of times they chose to delay for the experimenter (other).

\section{Procedure}

Children were tested in a single 45 -min session that included other cognitive tasks not reported in this article. All procedures were approved by the research ethics boards at the University of Oregon and University of Ottawa.

\section{Results and discussion}

There were no effects of gender, reward type (candies, stickers, or nickels), size of the delayed reward (two, four, or six later), or the order of questions (self or other first), so these factors were not included in the subsequent analysis.

A 5 (Age Group: 3-, 4-, 5-, 6-, or 7-year-olds) by 2 (Perspective: self or other) repeated-measures analysis of variance (ANOVA) on choice delay performance revealed a main effect of age, $F(4,86)=8.69, p<.001, \eta_{\mathrm{p}}^{2}=.288$. Tukey post hoc comparisons showed that this effect was driven by 6 - and 7-year-olds outperforming 3 - and 4 -year-olds ( $p s<.02$ ). There was no significant effect of perspective $\left(p=.82, \eta_{\mathrm{p}}^{2}=.001\right)$. There was, however, a significant interaction between age group and perspective, $F(4,86)=7.15, p<.001, \eta_{\mathrm{p}}^{2}=.250$. The 3-year-olds benefitted from taking the other's perspective $(M=4.85, S D=3.13)$ compared with their own perspective $(M=2.60, S D=2.46)$, $t(19)=4.27, p<.001$, Cohen's $d=0.95,95 \%$ confidence interval $(\mathrm{CI})[-3.35,-1.15]$. In contrast, the 6 -year-olds benefitted from taking their own perspective $(M=6.84, S D=2.17)$ compared with the other's perspective $(M=5.10, S D=2.13), t(18)=2.37, p=.03$, Cohen's $d=0.55,95 \%$ CI $[0.20,3.28]$, and the 7-year-olds marginally benefitted, $t(17)=2.02, p=.06$, Cohen's $d=0.47,95 \% \mathrm{CI}[-0.04$, 1.93] (see Fig. 1). The 4- and 5-year-olds did not perform differently for themselves versus other ( $p$ s > .09, Cohen's $d s<0.39$ ).

Similar to Prencipe and Zelazo (2005) results, 3-year-olds were better at delaying for the experimenter than for themselves, whereas 4- and 5-year-olds delayed for themselves just as often as they

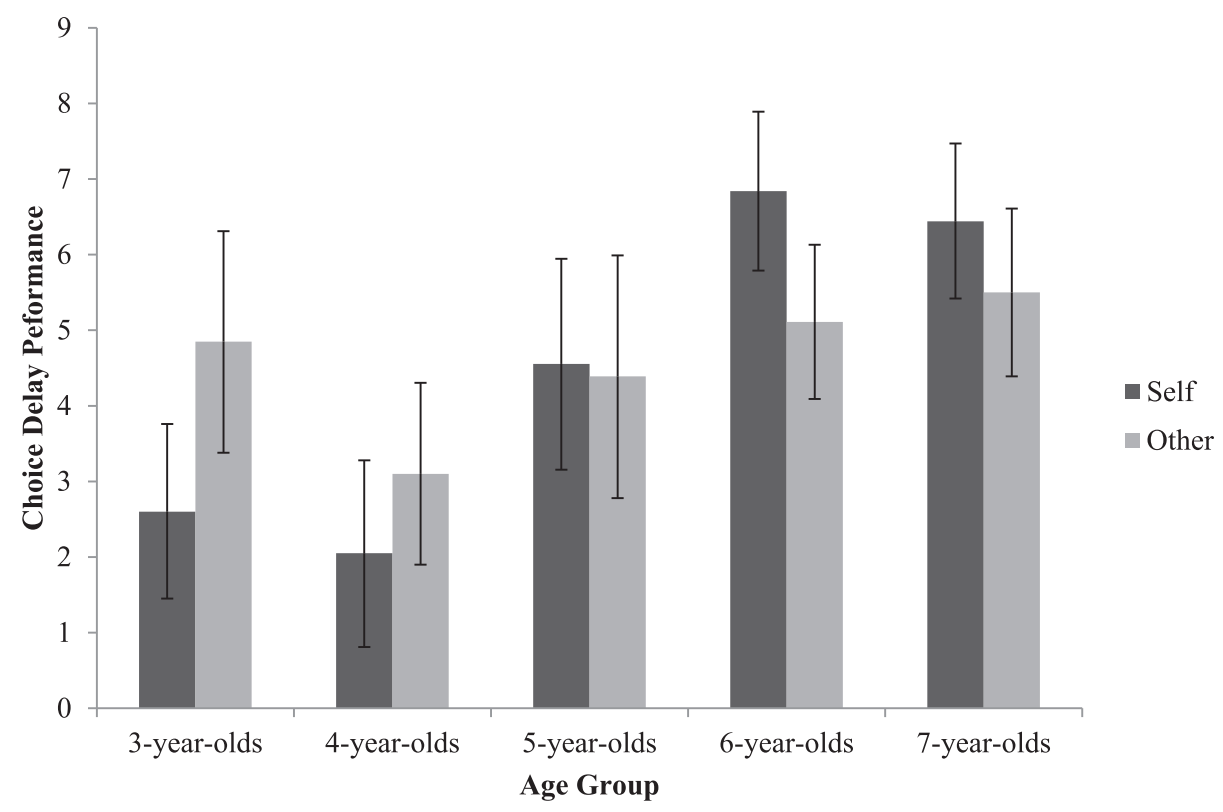

Fig. 1. Experiment 1 performance on choice delay task by age group and perspective. Error bars show $95 \%$ confidence intervals. 
delayed for the experimenter. Our results also extend Prencipe and Zelazo's results by showing that 6- and 7-year-olds tended to perform better for self than for other. Perhaps by 6 and 7 years of age, motivation becomes a helpful factor in delaying for a larger reward for the self-in contrast to the hindering factor it seems to be with 3-year-olds, who appear to be incapable of distancing themselves from their current desire for gratification. Older children may also be developing a greater appreciation of the experimenter's motivation (i.e., that the other person might also desire rewards immediately) with greater integration of first- and third-person perspectives (Barresi \& Moore, 1996; Prencipe \& Zelazo, 2005). This finding suggests that, prior to 4 years of age, delaying gratification for another person is easier than delaying gratification for the self and also that children seem to increasingly take a third-person perspective on the self and a first-person perspective on another person as they age (Prencipe \& Zelazo, 2005).

Although the self-other differences that we obtained varied across age groups, younger children benefitted from adopting another person's perspective. Children may reason more optimally about another person's future, as compared with their own future, because doing so creates "psychological distance," thereby minimizing conflict between children's current and future desires or states. Relatedly, young children's executive control benefits from taking a more detached "exemplar" perspective or a third-person perspective compared with a first-person perspective (White \& Carlson, 2016).

\section{Experiment 2}

Another way to manipulate psychological distance is through the use of language terms that might prime a more impulsive immediate decision versus a more rational delayed decision. Specifically, use of the auxiliary verb "should" might encourage children to distance themselves from their current desires and reflect on more optimal decisions, whereas the auxiliary verb "want" might prime children to make an impulsive decision based on immediate desires. Given past research suggesting that children's DoG is influenced by group norms (Doebel \& Munakata, 2018) as well as cultural socialization goals (Lamm et al., 2018), it is possible that using the word "should" primes children to respond in a more optimal manner, resulting in superior DoG performance for self and other. In contrast, using the word "want" might prime children to focus on their current desires and, thus, could result in worse DoG performance for both self and other.

In addition, one limitation of Experiment 1, and indeed of Prencipe and Zelazo (2005) original study, is that children were asked slightly different questions when delaying gratification for self and other. Children were asked "What do you want to do" when making DoG choices for themselves, whereas they were asked "What should I do?" when making DoG choices for the experimenter. It is possible that these differences in wording, rather than differences in perspective (i.e., self vs. other), led to the self-other differences observed in Experiment 1. Therefore, the main goals of Experiment 2 were to replicate the findings of Experiment 1 and also to examine the impact of the auxiliary verbs "want" and "should" on children's DoG choices for self and other to explore the role that language might play in psychological distancing.

\section{Method}

\section{Participants}

A total of 143 children participated in the experiment, but 11 were excluded due to their parents not allowing candy to be offered during relevant candy trials $(n=4)$, a diagnosis of autism spectrum disorder $(n=3)$, experimenter error $(n=3)$, or failure to report the child's date of birth $(n=1)$. The final sample consisted of 132 typically developing participants: 24 3-year-olds (8 girls; $M_{\text {age }}=41.17$ mon ths, $S D=3.32$ ), 284 -year-olds ( 16 girls; $M_{\text {age }}=53.54$ months, $S D=3.36$ ), 24 5-year-olds (11 girls; $M_{\text {age }}=64.17$ months, $\left.S D=3.42\right), 296$-year-olds $\left(15\right.$ girls; $M_{\text {age }}=77.83$ months, $S D=3.45$ ), and 27 7-year-olds ( 9 girls; $M_{\text {age }}=89.04$ months, $S D=3.12$ ). An a priori power analysis revealed that, based on a medium effect size (a conservative estimate based on Experiment 1 ) for the interaction between age group and perspective $(f=.25, \alpha=.05$, power $=.80$, correlation between repeated measures based on Experiment 1 results $=.53$ ), a sample size of 55 would be necessary. Thus, the current sample size 
had ample power to detect a similar-sized interaction effect as Experiment 1, but we tested a larger sample size due to a commitment to test in a museum setting (see below) during May to August 2019. Children were mostly White (59\% White, 4\% Asian, 2\% Middle Eastern, 8\% European, 8\% mixed heritage, $3 \%$ other, and $16 \%$ unknown) and from upper-middle-class backgrounds (70\% of parents held a university degree and $74 \%$ had a family income over $\$ 80,000$ ). Children were recruited from a participant database at the University of Ottawa and from a local science museum.

\section{Choice delay}

The choice delay task was identical to that in Experiment 1 except for one key difference: Children were randomly assigned to either a "should" condition or a "want" condition (between participants) within age groups. There were no significant differences across conditions in children's age and gender, $t s(130)<0.29, p s>.77$. Children in the "should" condition were asked what they should do for themselves (self condition: "What should you do?") and what the experimenter should do (other condition: "What should I do?") for all choices they made. In contrast, children in the "want" condition were asked what they wanted to do for themselves (self condition: "What do you want to do?") and what the experimenter wanted to do (other condition: "What do I want to do?") for all choices they made. Self and other blocks were counterbalanced; half of the children received the self block followed by the other block, and half received the other block followed by the self block.

\section{Procedure}

Children were tested in a single 15-min session. Children were tested either in the laboratory (40\%) or at the science museum (60\%). All procedures were approved by the research ethics boards at the Brock University and the University of Ottawa.

\section{Results and discussion}

There were no effects of gender, size of the delayed reward (two, four, or six rewards later), the order of questions (self or other block first), or testing location (laboratory or science museum), so these factors were not included in subsequent analyses. There was a main effect of reward type, $F$ $(2,244)=3.07, p=.048, \eta_{\mathrm{p}}^{2}=.03$, and reward type was involved in complex interactions (a twoway interaction with age group and a three-way interaction with age group and perspective). However, because it was not a main variable of interest (and thus not planned in our analysis), and because it did not affect the basic patterns of findings, it was not included in our main analysis.

A 5 (Age Group: 3-, 4-, 5-, 6-, or 7-year-olds) by 2 (Perspective: self or other) by 2 (Auxiliary Verb: want or should) repeated-measures ANOVA on choice delay performance revealed a significant main effect of age group, $F(4,122)=5.65, p<.001, \eta_{\mathrm{p}}^{2}=.16$. Tukey's post hoc comparisons showed that the effect of age was again driven by 6 - and 7-year-olds outperforming both 3 - and 4-year-olds ( $p s<.03$, Cohen's $d s>.75)$. Neither perspective, $F(1,122)=1.21, p=.27, \eta_{\mathrm{p}}^{2}=.01$, nor auxiliary verb, $F(1,122)=0.26, p=.61, \eta_{\mathrm{p}}^{2}=.002$, had a significant effect on DoG performance. Although nonsignificant, children's performance on "should" trials was numerically higher (self: $M=5.47, S D=3.37$; other: $M=5.20, S D=2.87$ ) than their performance on "want" trials (self: $M=5.40, S D=3.52$; other: $M=5.01, S D=3.07$ ). There was a marginal interaction between age group and perspective, $F(4,122)=2.29, p=.06, \eta_{\mathrm{p}}^{2}=.07$, which was driven by 3 -year-olds tending to perform better for other $(M=4.38, S D=3.03)$ compared with self $(M=3.25, S D=3.81), t(23)=2.01, p=.06$, Cohen's $d=0.41$, $95 \% \mathrm{CI}[-2.28,0.032]$, and 7-year-olds performing better for self $(M=6.96, S D=2.50)$ compared with other $(M=5.85, S D=2.40), t(26)=2.12, p=.04$, Cohen's $d=0.41,95 \% \mathrm{CI}[0.03,2.19])$. Fig. 2 shows DoG performance by age group and perspective. There were no differences between performance for self and performance for other for 4-, 5-, and 6-year-olds ( $p s>.09$, Cohen's $d s<0.36$ ). No other interactions were significant.

Experiment 2 showed that 6- and 7-year-olds outperformed 3- and 4-year-olds on the DoG task, suggesting that substantial development in this domain occurs between 3 and 7 years of age and lending support for an extended developmental trajectory of DoG beyond 3 or 4 years.

The marginal interaction between age group and perspective was driven by 3 -year-olds tending to show an other-over-self advantage, whereas 7-year-olds showed the opposite pattern by performing 


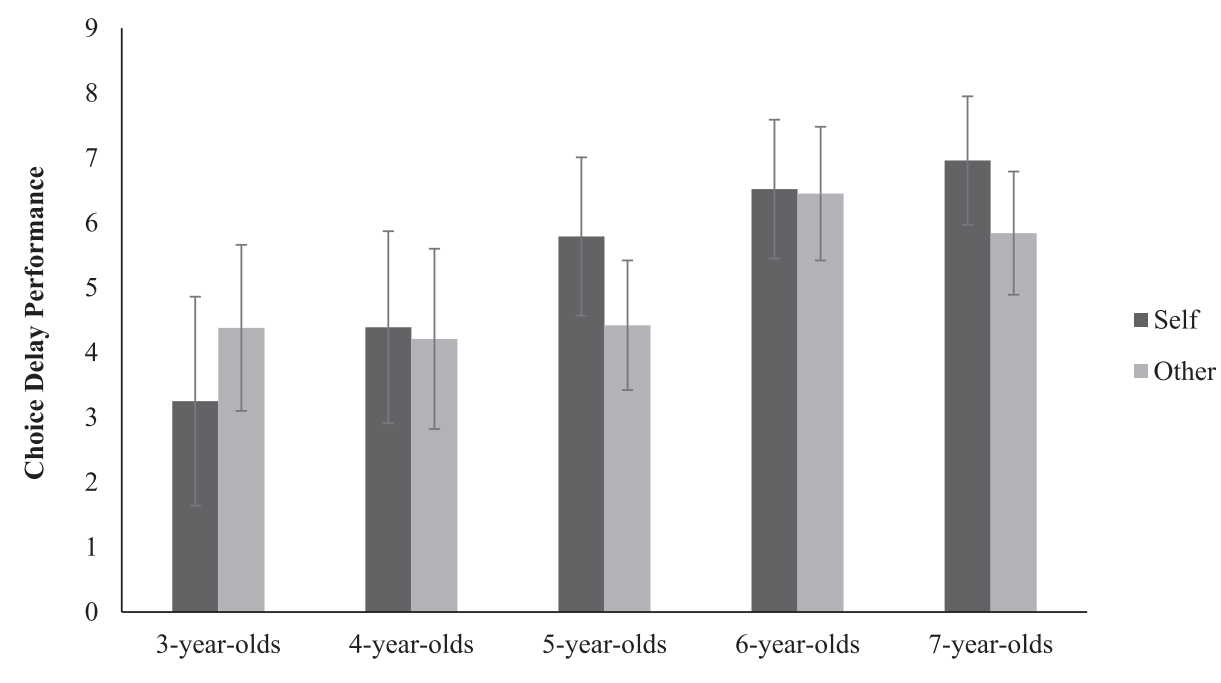

Age Group

Fig. 2. Experiment 2 performance on choice delay task by age group and perspective. Error bars show $95 \%$ confidence intervals.

better for themselves than for the experimenter. Thus, these results support Prencipe and Zelazo (2005) findings with 3-year-olds and also our Experiment 1 results with this age group. Our results also support the idea that 7-year-olds take more of a third-person perspective on the self and may also realize that the experimenter sometimes wants rewards immediately rather than delaying (i.e., taking a first-person perspective for other).

With respect to the main goal of Experiment 2, we found no effect of auxiliary verb type on DoG performance. That is, children performed similarly when they were asked what they or the experimenter "wanted" to do versus what they or the experimenter "should" do. Perhaps varying the auxiliary verb was too subtle a manipulation to affect children's DoG performance. More specifically, using the auxiliary verb "should" might not have been powerful enough to induce children to distance themselves from their current desires. Importantly, however, we can be reassured that self-other differences from Experiment 1 and from Prencipe and Zelazo (2005) original study were likely not due to the use of different auxiliary verbs in the questions "What do you want to do?" for themselves and "What should I do?" for the experimenter. Unlike other manipulations of social norms (e.g., Doebel \& Munakata, 2018), our auxiliary verb manipulation did not appear to influence psychological distance and did not affect children's delay choices.

\section{General discussion}

Taken together, the results of our two experiments suggest that DoG development extends beyond the preschool period and interacts with perspective in theoretically relevant ways. Whereas younger children (3-year-olds) make more delayed decisions for another person compared with themselves, older children (6- and 7-year-olds) tend to delay more for themselves than for the experimenter. The 3-year-olds might be heavily influenced by their current desires and, thus, unable to overcome their current state to accurately think about the future for themselves (Atance \& Meltzoff, 2006; Mahy, 2016), but they seem to make more optimal delay choices for an adult experimenter. By 6 or 7 years of age, however, children seem able to take more of a third-person perspective on the self (i.e., realizing that delaying for a larger reward is the better choice) as well as a first-person perspective on another person's decision making, realizing that other people might sometimes want a small reward immediately rather than waiting for a larger reward (Barresi \& Moore, 1996; Prencipe \& 
Zelazo, 2005). It may be that older children are developing a stronger connection with their future selves, in line with the future self-continuity hypothesis (Hershfield, 2011) that might allow them to make wiser future decisions for themselves than do younger children.

However, later in childhood and into adulthood, the other-over-self advantage seems to reemerge in some domains of decision making (e.g., Kross \& Grossmann, 2012). For example, adults make wiser decisions when reasoning about another person's problems compared with their own problems (Grossmann \& Kross, 2014; Kross \& Grossmann, 2012), and self-distancing reduces this self-other asymmetry, suggesting that taking a more detached perspective from the self promotes superior reasoning. Furthermore, adults predict that another person's future preferences will change more than their own (Bauckham et al., 2019; Renoult, Kopp, Davidson, Taler, \& Atance, 2016), and they are less impulsive in delay discounting tasks when the other individual is not a family member and, thus, is a more distant rather than close other (Ziegler \& Tunney, 2012). How can this be explained? It is possible that DoG paradigms might show different developmental patterns than the more hypothetical or preference-based tasks used to measure self-other asymmetry in adult samples. Specifically, children might show a decrease in self-other differences as they begin to appreciate others' desires as well as understand what decisions are in their best interest (delaying for a larger reward), whereas adults might show an other-over-self advantage in reasoning about hypothetical situations because they are able to take a more distanced perspective (by inhibiting others' desires or preferences). Because selfother differences have been studied using different tasks and age groups, an important future direction is to more systematically examine this asymmetry across the lifespan while using identical or highly similar tasks.

Increasing psychological distance by manipulating perspective was more effective than manipulating auxiliary verbs to prime a more rational (use of "should") versus impulsive (use of "want") self. This is an important finding given that past work has used "should" when asking children about the experimenter and "want" when asking children about their own delay choices (our Experiment 1 ; Prencipe \& Zelazo, 2005). Thus, the finding that auxiliary verbs do not affect children's performance strengthens our conclusions that it is specifically perspective (i.e., self or other) that interacts with age to affect children's DoG choices. Perhaps the auxiliary verb manipulation employed in Experiment 2 would be effective in an adult sample, but not in a sample of young children who might not be as sensitive as adults to the difference in meaning between "want" and "should" (although children seem to understand these terms fairly early in development; e.g., Cummins, 1996; Harris \& Nunez, 1996).

One limitation of the current work is that the other in both experiments was an adult experimenter rather than a same-aged peer. Therefore, it is possible that 3-year-olds' DoG was better for the other because they viewed these children experimenter as "wiser" and not because of psychological distance between self and other. However, the findings with 6- and 7-year-olds are at odds with this interpretation unless by this age children have realized that adults do not always wish to make the wiser choice and sometimes want a smaller immediate reward. Moreover, the other-over-self advantage has been shown in episodic foresight tasks when decisions are made for a same-aged peer rather than an adult (Bélanger et al., 2014; Russell et al., 2010), suggesting that our results are not driven by young children simply viewing adults as wiser decision makers. Nonetheless, future work should replicate our results when the other is a same-aged peer. Other important future directions are to determine the extent to which self-other differences in DoG are related to self-other differences in other kinds of future-oriented reasoning tasks (e.g., Bélanger et al., 2014; Russell et al., 2010) and how self-other differences are related to self-referential processing more broadly.

Our study is the first to systematically examine how children's DoG is affected by adopting a self versus other perspective across a relatively wide age range. We replicated Prencipe and Zelazo (2005) findings with 3- and 4-year-olds across two large experiments and also showed that the effects of perspective (i.e., self vs. other) interact with age in theoretically relevant ways. For example, whereas adopting the perspective of another may benefit younger preschoolers' DoG decisions, older children show the reverse effect (i.e., performance for self is better than performance for other). Although the interaction between age and perspective was only marginally significant in Experiment 2, it was in the expected direction and, together with Experiment 1, supports the claim that age and perspective interact in important ways to affect children's DoG. 
In sum, the current experiments suggest that children's DoG improves beyond the preschool period and that younger children's (3-year-olds) DoG performance is more likely to benefit from increased psychological distance compared with older children's (6- and 7-year-olds) DoG performance, with the older children seeming to perform more optimally for themselves than for the experimenter. Manipulating normative (should) or preference-based (want) language had no impact on performance, suggesting that this might be a less effective way to manipulate psychological distance with young children. Future work should continue to examine the effects of psychological distance in other domains of children's future-oriented decision making and in their sociocognitive development more broadly.

\section{Acknowledgments}

Preparation of the manuscript was funded partly by Natural Sciences and Engineering Research Council of Canada (NSERC) Discovery Grants to C.E.V.M. (RGPIN-2015-03774) and C.M.A. (RGPIN2015-06446). We thank Michele Anderson, Prachi Bhuptani, Jack Rossing, and Madeline Weissman for assistance with data collection as well as the families and children who participated.

\section{Appendix A. Supplementary material}

Supplementary data to this article can be found online at https://doi.org/10.1016/j.jecp.2019. 104767.

\section{References}

Atance, C. M., \& Meltzoff, A. N. (2006). Preschoolers' current desires warp their choices for the future. Psychological Science, 17, $583-587$.

Barresi, J., \& Moore, C. (1996). Intentional relations and social understanding. Behavioral and Brain Sciences, 19, $107-121$.

Bauckham, G., Lambert, R., Atance, C. M., Davidson, P. S., Taler, V., \& Renoult, L. (2019). Predicting our own and others' future preferences: The role of social distance. Quarterly Journal of Experimental Psychology (Hove), 72, 634-642.

Bélanger, M. J., Atance, C. M., Varghese, A. L., Nguyen, V., \& Vendetti, C. (2014). What will I like best when I'm all grown up? Preschoolers' understanding of future preferences. Child Development, 85, 2419-2431.

Cassidy, K. W. (1998). Three- and four-year-old children's ability to use desire- and belief-based reasoning. Cognition, 66, B1-B11.

Cummins, D. D. (1996). Evidence of deontic reasoning in 3- and 4-year-old children. Memory \& Cognition, 24, 823-829.

Doebel, S., \& Munakata, Y. (2018). Group influences on engaging self-control: Children delay gratification and value it more when their in-group delays and their out-group doesn't. Psychological Science, 29, 738-748.

Grossmann, I., \& Kross, E. (2014). Exploring “Solomon's paradox": Self-distancing eliminates the self-other asymmetry in wise reasoning about close relationships in younger and older adults. Psychological Science, 25, 1571-1580.

Harris, P. L., \& Nunez, M. (1996). Understanding of permission rules by preschool children. Child Development, 67, $1572-1591$.

Hershfield, H. E. (2011). Future self-continuity: How conceptions of the future self transform intertemporal choice. Annals of the New York Academy of Sciences, 1235, 30-43.

Kesek, A., Cunningham, W. A., Packer, D. J., \& Zelazo, P. D. (2011). Indirect goal priming is more powerful than explicit instruction in children. Developmental Science, 14, 944-948.

Kidd, C., Palmeri, H., \& Aslin, R. N. (2013). Rational snacking: Young children's decision-making on the marshmallow task is moderated by beliefs about environmental reliability. Cognition, 126, 109-114.

Kramer, H. J., Goldfarb, D., Tashjian, S. M., \& Lagattuta, K. H. (2017). "These pretzels are making me thirsty": Older children and adults struggle with induced-state episodic foresight. Child Development, 88, 1554-1562.

Kross, E., \& Grossmann, I. (2012). Boosting wisdom: Distance from the self enhances wise reasoning, attitudes, and behavior. Journal of Experimental Psychology: General, 141, 43-48.

Lamm, B., Keller, H., Teiser, J., Gudi, H., Yovsi, R. D., Freitag, C., ... Vöhringer, I. (2018). Waiting for the second treat: Developing culture-specific modes of self-regulation. Child Development, 89, e261-e277.

Lee, W. S., \& Atance, C. M. (2016). The effect of psychological distance on children's reasoning about future preferences. PLoS One, 11(10) e164382.

Lemmon, K., \& Moore, C. (2007). The development of prudence in the face of varying future rewards. Developmental Science, 10 , 502-511.

Mahy, C. E. V. (2016). Young children have difficulty predicting future preferences in the presence of a conflicting physiological state. Infant and Child Development, 25, 325-338.

Mazachowsky, T. R., Koktavy, C., \& Mahy, C. E. V. (2019). The effect of psychological distance on young children's future predictions. Infant and Child Development, 28(4) e2133.

Michaelson, L. E., \& Munakata, Y. (2016). Trust matters: Seeing how an adult treats another person influences preschoolers' willingness to delay gratification. Developmental Science, 19, 1011-1019.

Mischel, W., \& Baker, N. (1975). Cognitive transformations of reward objects through instructions. Journal of Personality and Social Psychology, 31, 254-261. 
Mischel, W., \& Ebbesen, E. B. (1970). Attention in delay of gratification. Journal of Personality and Social Psychology, 16, 329-337.

Mischel, W., Ebbesen, E. B., \& Zeiss, A. R. (1972). Cognitive and attentional mechanisms in delay of gratification. Journal of Personality and Social Psychology, 21, 204-218.

Mischel, W., \& Moore, B. (1973). Effects of attention to symbolically-presented rewards upon self-control. Journal of Personality and Social Psychology, 28, 172-179.

Mischel, W., Shoda, Y., \& Rodriguez, M. I. (1989). Delay of gratification in children. Science, 244, 933-938.

Moore, C., Barresi, J., \& Thompson, C. (1998). The cognitive basis of future-oriented prosocial behavior. Social Development, 7, $198-218$.

Prencipe, A., \& Zelazo, P. D. (2005). Development of affective decision making for self and other: Evidence for the integration of first- and third-person perspectives. Psychological Science, 16, 501-505.

Renoult, L., Kopp, L., Davidson, P. S., Taler, V., \& Atance, C. M. (2016). You'll change more than I will: Adults' predictions about their own and others' future preferences. Quarterly Journal of Experimental Psychology (Hove), 69, 299-309.

Russell, J., Alexis, D., \& Clayton, N. (2010). Episodic future thinking in 3- to 5-year-old children: The ability to think of what will be needed from a different point of view. Cognition, 114, 56-71.

Watts, T. W., Duncan, G. J., \& Quan, H. (2018). Revisiting the marshmallow test: A conceptual replication investigating links between early delay of gratification and later outcomes. Psychological Science, 29, 1159-1177.

Weisberg, D. P., \& Beck, S. R. (2010). Children's thinking about their own and others' regret and relief. Journal of Experimental Child Psychology, 106, 184-191.

Weisberg, D. P., \& Beck, S. R. (2012). The development of children's regret and relief. Cognition \& Emotion, 26, 820-835.

Wellman, H. M., \& Liu, D. (2004). Scaling of theory-of-mind tasks. Child Development, 75, 523-541.

Wellman, H. M., \& Miller, J. G. (2008). Including deontic reasoning as fundamental to theory of mind. Human Development, 51 , 105-135.

White, R. E., \& Carlson, S. M. (2016). What would Batman do? Self distancing improves executive function in young children. Developmental Science, 19, 419-426.

White, R. E., Prager, E. O., Schaefer, C., Kross, E., Duckworth, A. L., \& Carlson, S. M. (2017). The "Batman effect”: Improving perseverance in young children. Child Development, 88, 1563-1571.

Zelazo, P. D., Carlson, S. M., \& Kesek, A. (2008). The development of executive function in childhood. In C. A. Nelson \& M. Luciana (Eds.), Handbook of developmental cognitive neuroscience (2nd ed., pp. 553-574). Cambridge, MA: MIT Press.

Ziegler, F. V., \& Tunney, R. J. (2012). Decisions for others become less impulsive the further away they are on the family tree. PLoS One, 7(11) e49479. 